\title{
A novel quantum field approach to photoexcited insulators
}

\author{
E. Klotins \\ Institute of Solid State Physics, University of Latvia, 8 Kengaraga str., Riga, LV-1063, Latvia \\ E-mail: klotins@cfi.lu.lv
}

Received March 9, 2016, published online May 25, 2016

\begin{abstract}
In order to predict optical properties of insulating materials under intensive laser excitation, we generalized methods of quantum electrodynamics, allowing us to simulate excitation of electrons and holes, interacting with each other and acoustic phonons. The prototypical model considers a two-band dielectric material characterized by the dispersion relations for electron and hole states. We developed a universal description of excited electrons, holes and acoustic phonons within joint quantum kinetics formalism. Illustrative solutions for the quasiparticle birth-annihilation operators, applicable at short laser pulses at $0 \mathrm{~K}$, are obtained by the transition from the macroscopic description to the quantum field formalism.
\end{abstract}

PACS: 79.20.Ds Laser-beam impact phenomena.

Keywords: insulating materials, laser excitation.

\section{Introduction}

Theoretical prediction of optical and transport properties of semiconductors and insulators under strong electromagnetic excitation, e.g. laser pulses, is quite chalenging problem. In these strongly correlated materials interactions between the charged particles play a major role in determining optical and transport properties: each single particle has a complex influence on its neighbors and interacts with both antiparticles (electrons-holes) as well as with crystalline phonons. The elaborated models based on the density functional theory (DFT) and the dynamical mean-field theory of strongly correlated fermion systems (DMFT) which now became the standard for fermionic correlation problems [1-3]. Nevertheless, the uncertainty in the choice of model parameters prevent the DFT+DMFT approaches from being flexible enough to capture entirely the quantum many-body problem of realistic complexity. Thus, new mathematical techniques are required for physical systems where perturbation techniques are not applicable.

A complementary alternative approach is the quantum electrodynamics (QED) as derived from the simplest approaches of quantum field theory based on the Dirac sea for electrons and holes and the Hilbert spaces. Applications of QED to analysis of light-matter interaction to a large extent are based on fundamentals developed by Schwinger [4], Schmidt [5] and the mathematical techniques [6-8], where the model system is characterized by macroscopic disper- sion relations for noninteracting electrons and holes (however, correlated in momentum and energy) [9-11].

The QED based mathematical framework includes construction of the joint wave function of conduction and valence states, harmonized with the macroscopic dispersion relations and the restrictions of special relativity, canonical quantization, the minimum action approach and, finally, the nonequilibrium distribution functions of electrons and holes excited as a result of interaction with electromagnetic radiation. This framework could be applied to the nonMarkovian quantum kinetics driven by appropriate short laser pulses.

The rationale of this QED framework is that it gives clues to actual problems in low-temperature physics associated, for example, with magnetization dynamics induced by femtosecond light pulses [12], electronic spectra [13] and the limitations of application of conventional models of strongly correlated electron systems [14].

A step beyond this nondissipative prototypical approach is interaction with acoustic phonons. In this manuscript the canonical quantization of electron-hole pairs is paralleled with a description of acoustic phonons based on well-known stationary solutions for displacement vector field $[15,16]$ and decomposed within the framework of joint quantum kinetics framework.

A systematic step-by-step quantum kinetic calculations are addressed to a typical two-band model of insulator and a model of linear elasticity, both fitting in the framework [10] 
for nonequilibrium distribution functions, as a basis for further advancements and improvements.

The manuscript is organized as follows:

In Sec. 2 the basic definitions and denominations are presented with a special emphasis on the time evolution of quasiparticle observables and their quantum kinetics.

Section 3 is addressed to the effect of electromagnetic field on the ensemble of electrons and holes, formalized in terms of gauge invariant minimal substitution [17] and complete relativistic quantum field approach [18] is provided for covariance under the full Poincaré group. The conditions of its use are large number of identical copies of the charged matter and the wavelength of optical radiation exceeding considerably the lattice constant.

In Sec. 4 the quantum field approach for electrons and holes is extended to the quantum kinetics of acoustic phonons, on the equal oscillatory equation basis as precondition to embody both the excitation and the relaxation of the quasiparticles.

In Sec. 5 the canonical quantization of electronic subsystem is extended to acoustic phonons on equal KleinGordon basis and consequences for the systems with weak and strong interaction outlined.

Conclusions are given in Sec. 6.

\section{Basic definitions and denominations}

This section is addressed to the transition from a macroscopic Hamiltonian system to its quantum field counterpart with a special emphasis on a system that is made up of two subsystems. In essence, a larger Hilbert space is constructed from the tensor product of the smaller sub-Hilbert spaces. It is the case of a compound system of conduction and valence states in two-band dielectric build up by tensor product of two-wave functions $\mathbb{H}=\mathbb{H}_{1} \otimes \mathbb{H}_{2}$ [19].

The free field electron-hole theory starts with classical Hamiltonian for single particle

$$
H=\mathbf{p}^{2} /(2 m)+V,
$$

where $\mathbf{p}$ is the quasi-momentum and the nonvanishing term $V$ is potential energy. Free field description of the system is given by the solutions of the equation $[H-E]|\Psi\rangle=0$ applied to $\mathrm{e}-\mathrm{h}$ pair in the conduction $(c)$ and valence $(v)$ bands

$$
E_{C}=\frac{\Delta}{2}+\varepsilon_{C}(\mathbf{p}), \quad E_{v}=-\frac{\Delta}{2}-\varepsilon_{v}(\mathbf{p}),
$$

where the dispersion laws of the kinetic energy $\varepsilon_{C}(\mathbf{p})$ and $\varepsilon_{v}(\mathbf{p})$ are determined by methods of nonrelativistic quantum mechanics. The general dispersion law is defined as [10]

$\left(E-E_{C}\right)\left(E-E_{v}\right)=\left[E-\frac{\Delta}{2}-\varepsilon_{c}(\mathbf{p})\right]\left[E+\left(\frac{\Delta}{2}\right)+\varepsilon_{v}(\mathbf{p})\right]=0$.
For quadratic dispersion and in the effective mass approximation, the general dispersion (3) becomes

$\left(E-E_{c}\right)\left(E-E_{v}\right)=\left[E-\frac{\Delta}{2}-\left(\frac{\mathbf{p}^{2}}{2 m_{e}}\right)\right]\left[E+\left(\frac{\Delta}{2}\right)+\left(\frac{\mathbf{p}^{2}}{2 m_{h}}\right)\right]=0$.

Here the band gap $\Delta$ and the band boundaries represented by dispersion laws $\varepsilon_{c, v}(\mathbf{p})$ remain invariable. Quantized free-field equation of motion (4) is given by the nonrelativistic energy relation in operator form

$$
\left[\hat{E}-\frac{\Delta}{2}-\varepsilon_{C}(\hat{\mathbf{p}})\right]\left[\hat{E}+\left(\frac{\Delta}{2}\right)+\varepsilon_{v}(\hat{\mathbf{p}})\right] \Psi(\mathbf{x}, t)=0,
$$

where $\Psi(\mathbf{x}, t)$ is the joint wave function of the two-particle states constituting a compound system equivalent to the two-particle Hilbert space $\mathbb{H}=\mathbb{H}_{1} \otimes \mathbb{H}_{2}$ [19] and $\varepsilon_{C}(\hat{\mathbf{p}})$ and $\varepsilon_{v}(\hat{\mathbf{p}})$ are gradient operators $\hat{\mathbf{p}} \rightarrow-i \hbar \nabla$ applied to unknown yet joint wave function. Replacement $\hat{E} \Rightarrow i \hbar \partial / \partial t$ in (5) results in kinetic equation transformed into uniform differential form of Klein-Gordon type with respect to time for the auxiliary operator function $\Phi(\mathbf{p}, t)$ [20]:

$$
\ddot{\Phi}(\mathbf{x}, t)+\Omega^{2}(\hat{\mathbf{p}}) \Phi(\mathbf{x}, t)=0
$$

with the effective frequency

$$
\Omega(\hat{\mathbf{p}})=\frac{\Delta+\varepsilon_{V}(\hat{\mathbf{p}})+\varepsilon_{C}(\hat{\mathbf{p}})}{2 \hbar} .
$$

The corresponding Lagrangian density for the auxiliary operator function $\Phi(\mathbf{x}, t)$ reads as

$$
\mathcal{L}(\mathbf{x}, t)=\frac{\partial}{\partial t} \Phi(\mathbf{x}, t) \frac{\partial}{\partial t} \Phi^{*}(\mathbf{x}, t)-\Omega^{2}(\hat{\mathbf{p}}) \Phi(\mathbf{x}, t) \Phi^{*}
$$

Going back to the total wave function (field function) $\Psi(\mathbf{x}, t)$ the Lagrangian density $\mathcal{L}$ reads as [20]

$$
\begin{gathered}
\mathcal{L}\left(\Psi, \Psi^{*} ; \dot{\Psi}, \dot{\Psi}^{*}\right)= \\
=\alpha\left\{\left[\frac{\left(\varepsilon_{C}(\hat{\mathbf{p}})-\varepsilon_{V}(\hat{\mathbf{p}})\right)^{2}}{4 \hbar^{2}} \Psi \Psi^{*}+\frac{i\left(\varepsilon_{C}(\hat{\mathbf{p}})-\varepsilon_{V}(\hat{\mathbf{p}})\right)}{2 \hbar} \Psi \dot{\Psi}^{*}-\right.\right. \\
\left.\left.-\frac{i\left(\varepsilon_{C}(\hat{\mathbf{p}})-\varepsilon_{V}(\hat{\mathbf{p}})\right)}{2 \hbar} \Psi^{*} \dot{\Psi}+\dot{\Psi} \dot{\Psi}^{*}\right]-\Omega^{2}(\hat{\mathbf{p}}) \Psi^{*} \Psi\right\} . \quad
\end{gathered}
$$

The prefactor $\alpha=1 / \Delta$ in Lagrangian density is based on a correspondence principle: at the band gap $\Delta \rightarrow \infty$ the Lagrangian density turns to zero [11]. Higher order terms in (9) are discarded. Derived quantities of the Lagrangian density are canonical momentum

$$
\pi(\mathbf{x}, t)=\frac{\partial \mathcal{L}}{\partial \dot{\Psi}}=\frac{1}{\Delta}\left(-\frac{i\left(\varepsilon_{c}(\hat{\mathbf{p}})-\varepsilon_{v}(\hat{\mathbf{p}})\right)}{2 \hbar} \Psi^{*}(\mathbf{x}, t)+\dot{\Psi}^{*}(\mathbf{x}, t)\right)
$$


and its complex conjugate (c.c). The free field Hamiltonian density (Hamiltonian function) reads as

$$
\mathbb{H}=\pi \dot{\Psi}+\pi^{*} \dot{\Psi}^{*}-\mathcal{L} .
$$

Relations (10), (11) constitute the basis for a subsequent kinetic description of electron-hole pairs.

\section{Lagrange-Hamilton approach for electron-hole pair}

The effect of electromagnetic field on the conduction and valence states is incorporated into classical Hamiltonian (1), through a gauge invariant minimal substitution $\mathbf{p} \rightarrow \mathbf{p}-q \mathbf{A}[17,21]$

$$
H=\frac{1}{2 m}[\mathbf{p}-q \mathbf{A}(\mathbf{x}, t)]^{2}+V,
$$

where $q$ is charge of the particle and the term $\mathbf{p}^{2} / 2 m$ is equal to the energy of a free particle. For an electron the charge $q_{e}=-|e|$ and for a hole $q_{h}=+|e|$ and $V$ is the potential energy associated with the band width. A restrictive presumption is the wavelength much larger than the lattice constant at which the nonstationary electric field $E=-\dot{\mathbf{A}}$ is regarded as spatially homogeneous and the terms $q^{2} \mathbf{A}^{2} / 2 m$ quadratic in $\mathbf{A}=\{0,0, A(t)\}$ are canceled. The Lagrangian density (9) now reads as

$$
\begin{gathered}
\mathcal{L}\left(\Psi, \Psi^{*} ; \dot{\Psi}, \dot{\Psi}^{*}\right)= \\
=\alpha\left\{\frac{\left(\left[\varepsilon_{c}(\hat{\mathbf{P}})-\varepsilon_{V}(\hat{\mathbf{P}})\right)^{2}\right.}{4 \hbar^{2}} \Psi \Psi^{*}+\frac{i\left(\varepsilon_{c}(\hat{\mathbf{P}})-\varepsilon_{V}(-\hat{\mathbf{P}})\right)}{2 \hbar} \Psi \dot{\Psi}^{* *}-\right. \\
\left.\left.-\frac{i\left(\varepsilon_{C}(\hat{\mathbf{P}})-\varepsilon_{V}(-\hat{\mathbf{P}})\right)}{2 \hbar} \Psi^{*} \dot{\Psi}+\dot{\Psi} \dot{\Psi}\right]-\Omega^{2}(\hat{\mathbf{P}}) \Psi^{*} \Psi\right\}, \quad \text { (13) }
\end{gathered}
$$

where the kinetic dispersions in operator representation are given by

$$
\begin{gathered}
\varepsilon_{C}(\hat{\mathbf{P}})=\frac{\hat{\mathbf{p}}^{2}}{2 m_{e}}+\frac{q \mathbf{A} \cdot \hat{\mathbf{p}}}{2 m_{e}}, \\
\varepsilon_{v}(-\hat{\mathbf{P}})=\frac{\hat{\mathbf{p}}^{2}}{2 m_{h}}-\frac{q \mathbf{A} \cdot \hat{\mathbf{p}}}{2 m_{h}} .
\end{gathered}
$$

Here $q \equiv|e|$ and we are working in the Coulomb gauge $\nabla \cdot \mathbf{A}=0$.

The Lorentz invariance of field functions implemented by four-Fourier transform with $\exp ( \pm \mathbf{p x} / \hbar)$-type coordinate depending terms that leads to the correspondence $\hat{\mathbf{p}} \rightarrow \mathbf{p}$ and $\hat{\mathbf{p}}^{2} \rightarrow \mathbf{p}^{2}$ and allows to simplify the gradient operators in (14), (15) as follows:

$$
\varepsilon_{C}(\hat{\mathbf{P}})=\varepsilon_{c}(\mathbf{p})+\frac{q \mathbf{A}(t) \cdot \mathbf{p}}{2 m_{e}},
$$

$$
\varepsilon_{v}(-\mathbf{P})=\frac{\mathbf{p}^{2}}{2 m_{h}}-\frac{q \mathbf{A} \cdot \mathbf{p}}{2 m_{h}}
$$

In view of (16), (17) the free field Lagrangian density (9) becomes time-dependent:

$$
\begin{gathered}
\mathcal{L}\left(\Psi, \Psi^{*} ; \dot{\Psi}, \dot{\Psi}^{*}\right)= \\
=\frac{1}{\Delta}\left\{\left[\frac{\left(\varepsilon_{c}(\mathbf{P})-\varepsilon_{V}(\mathbf{P})\right)^{2}}{4 \hbar^{2}} \Psi \Psi^{*}+\frac{i\left(\varepsilon_{c}(\mathbf{P})-\varepsilon_{V}(-\mathbf{P})\right)}{2 \hbar} \Psi \dot{\Psi}^{*}-\right.\right. \\
\left.\left.-\frac{i\left(\varepsilon_{c}(\mathbf{P})-\varepsilon_{V}(-\mathbf{P})\right)}{2 \hbar} \Psi^{*} \dot{\Psi}+\dot{\Psi} \dot{\Psi}^{*}\right]-\Omega^{2}(\mathbf{P}) \Psi^{*} \Psi\right\} \cdot(18)
\end{gathered}
$$

Similarly, the free field canonical momenta (10) transforms in

$\pi(\mathbf{x}, t)=\frac{\partial \mathcal{L}}{\partial \dot{\Psi}}=\frac{1}{\Delta}\left[-i \frac{\varepsilon_{c}(\mathbf{P})-\varepsilon_{v}(-\mathbf{P})}{2 \hbar} \Psi^{*}(\mathbf{x}, t)+\dot{\Psi}^{*}(\mathbf{x}, t)\right]$

and the c.c. of (19).

\section{Quantum field functions for electron-hole pair}

In this section the Lorentz invariant quantum field function in (18) is obtained by four-Fourier transform related to electron

$$
\Psi(x)=\int \frac{d^{4} p}{(2 \pi)^{4}} \tilde{\Psi}(p) \exp \left(-i \frac{p x}{\hbar}\right)
$$

and the c.c. (20) related to holes [18]. Properties of the system are implemented in the momentum space field $\tilde{\Psi}(p)$ and $p x=\frac{E t}{\hbar}-\frac{\mathbf{p} \cdot \mathbf{x}}{\hbar}$ is four-vector scalar product in $\{+,-,-,-\}$ metric signature.

Decomposition of the field functions is case specific and is determined by the condition of decomposition. For the electron-hole problem the momentum space field in (35) and c.c. of (20) yield

$$
\begin{gathered}
\tilde{\Psi}(E, \mathbf{p})=\delta\left[E-\frac{\Delta}{2}-\varepsilon_{C}(\mathbf{P})\right]\left[E+\frac{\Delta}{2}+\varepsilon_{V}(-\mathbf{P})\right] \psi(E, \mathbf{p})= \\
=\delta(g(E, \mathbf{p})) \psi(E, \mathbf{p})
\end{gathered}
$$

and the c.c. of (21). The field functions are obtained by implementing relations (21) into the four-Fourier transforms

$\Psi(\mathbf{x}, t)=\int \frac{d p_{1} d p_{2} d p_{3}}{(2 \pi)^{3}}\left(\int \frac{d E}{2 \pi} \delta(g(E, \mathbf{p})) f(E, \mathbf{p})\right) \mathrm{e}^{i \frac{\mathbf{p x}}{\hbar}}$

and the c.c. of (22). 
The $f$-functions read as

$$
f(E, \mathbf{p})=\psi(E, \mathbf{p}) \mathrm{e}^{-i \frac{E t}{\hbar}}
$$

and c.c. of (23). As a result, the problem of finding the field functions (22) is reduced in two integrals over momentum $p_{0}$ with integrands comprising delta functions obeying the integral forms of the generalized scaling property

$$
\int_{-\infty}^{\infty} f(E, \mathbf{p}) \delta(g(E, \mathbf{p})) d E=\sum_{i} \frac{f\left(E_{i}, \mathbf{p}\right)}{\left|g^{\prime}\left(E_{i}\right)\right|},
$$

where the condition of decomposition is determined by the $g$-function (3)

$$
g(E, \mathbf{p}, t)=\left[E-\frac{\Delta}{2}-\varepsilon_{C}(\mathbf{p}, t)\right]\left[E+\frac{\Delta}{2}+\varepsilon_{v}(-\mathbf{p}, t)\right] .
$$

By simple algebra $[11,20]$ the direct and complex conjugate quantum field functions acquire integral representation with parameters of the macroscopic dispersion relations maintained:

$$
\begin{gathered}
\Psi(\mathbf{x}, t)= \\
=\int \frac{d \mathbf{p}}{(2 \pi)^{3}}\left(\frac { 1 } { 2 \hbar \Omega ( \mathbf { P } ) } \left(\psi\left(\Delta / 2+\varepsilon_{c}(\mathbf{P})\right) \mathrm{e}^{-i\left(\frac{\Delta / 2+\varepsilon_{c}(\mathbf{P})}{\hbar}\right) t}+\right.\right. \\
\left.+\psi\left(-\Delta / 2-\varepsilon_{v}(-\mathbf{P})\right) \mathrm{e}^{i\left(\frac{\Delta / 2+\varepsilon_{v}(-\mathbf{P})}{\hbar}\right) t}\right) \mathrm{e}^{\left.i \frac{\mathbf{p x}}{\hbar}\right)}
\end{gathered}
$$

and the c.c. of (26).

In (26) the effective frequency contributes to the relativistic invariant integration measure [17] as

$$
\frac{d \mathbf{p}}{(2 \pi)^{3}} \frac{1}{2(\hbar \Omega(\mathbf{P}))} \text {. }
$$

In the relations above the time varying optical radiation is formally implemented as $\varepsilon_{c}(\mathbf{P}(\mathbf{p}, t)) \Rightarrow \varepsilon_{C}(\mathbf{p}, t)$, $\varepsilon_{v}(\mathbf{P}(-\mathbf{p}, t)) \Rightarrow \varepsilon_{v}(-\mathbf{p}, t)$ (16). Complete relativistic quantum field approach [19] is provided for covariance under the full Poincaré group. To this end the relativistic invariant integration measure (27) is added with invariant normalization for states given by factor $(2 \pi)^{3 / 2} \sqrt{2 \hbar \Omega(\mathbf{P})}$. Another item is transition from the infinite-volume limit (26) to the Big Box representation of field functions. With periodic boundary conditions in the box of volume $L^{3}$ the momentum $\mathbf{p}=\hbar \mathbf{k}$ is given by $p_{j}=\hbar 2 \pi n_{j} / L$, $n_{j}=0, \pm 1, \pm 2 \ldots, \pm \infty, j=x, y, z$ and the quantization volume is given by $d \mathbf{p}=(\hbar 2 \pi / L)^{3}$. Finally, the factor to normalize field amplitudes is given by redefinition $a(\mathbf{p})=(L / 2 \pi)^{3 / 2} a_{\mathbf{p}}$.
These ingredients, put together, turns to the prefactor to field functions as $\frac{1}{\sqrt{V}} \frac{\sqrt{\Delta}}{\sqrt{2 \hbar \Omega(\mathbf{P})}}$. The renormalized field functions (26) read as

$$
\begin{aligned}
\Psi(\mathbf{x}, t)= & \frac{1}{\sqrt{V}} \sum_{\mathbf{p}} \frac{\sqrt{\Delta}}{\sqrt{2 \hbar \Omega(\mathbf{P})}}\left(\psi\left(\Delta / 2+\varepsilon_{c}(\mathbf{p})\right) \mathrm{e}^{-i\left(\frac{\Delta / 2+\varepsilon_{c}(\mathbf{p})}{\hbar}\right) t}+\right. \\
& \left.+\psi\left(-\Delta / 2-\varepsilon_{V}(-\mathbf{p})\right) \mathrm{e}^{i\left(\frac{\Delta / 2+\varepsilon_{v}(-\mathbf{p})}{\hbar}\right) t}\right) \mathrm{e}^{i \frac{\mathbf{p x}}{\hbar}}
\end{aligned}
$$

and the c.c. of (28); the impact of radiation is maintained exclusively in the effective frequency.

The quasiparticle representation is based on action integral $S=\int d x \mathcal{L}$ where the Lagrangian density is connected with Hamiltonian density by relation (11):

$$
S=\int d x\left\{\pi \dot{\Psi}+\pi^{*} \dot{\Psi}^{*}-\mathbb{H}\right\}
$$

The necessary operator representation of field functions (28) is obtained by replacements

$$
\begin{gathered}
\psi\left(\Delta / 2+\varepsilon_{c}(\mathbf{p})\right) \Rightarrow \hat{a}_{e}(\mathbf{p}), \psi\left(-\Delta / 2-\varepsilon_{v}(-\mathbf{p})\right) \Rightarrow \hat{a}_{h}(-\mathbf{p}), \\
\psi^{*}\left(\Delta / 2+\varepsilon_{c}(\mathbf{p})\right) \Rightarrow \hat{a}_{e}^{+}(\mathbf{p}), \psi^{*}\left(-\Delta / 2-\varepsilon_{v}(-\mathbf{p})\right) \Rightarrow \hat{a}_{h}^{+}(-\mathbf{p}),
\end{gathered}
$$

where the sub-indices for conduction and valence states in the dispersions $\varepsilon_{c, v}(\mathbf{p}, t)$ are exchanged by quasielectrons and holes in the field amplitudes $a_{e, h}(\mathbf{p}, t)$. The corresponding field operators read as

$$
\begin{aligned}
\hat{\Psi}(\mathbf{x}, t)= & \frac{1}{\sqrt{V}} \sum_{\mathbf{p}} \frac{\sqrt{\Delta}}{\sqrt{2 \hbar \Omega(\mathbf{p}, t)}}\left(\hat{a}_{e}(\mathbf{p}) \mathrm{e}^{-i\left(\frac{\Delta / 2+\varepsilon_{c}(\mathbf{p})}{\hbar}\right) t}+\right. \\
& \left.+\hat{a}_{h}(-\mathbf{p}) \mathrm{e}^{i\left(\frac{\Delta / 2+\varepsilon_{v}(-\mathbf{p})}{\hbar}\right) t}\right) \mathrm{e}^{i \frac{\mathbf{p} \mathbf{x}}{\hbar}}
\end{aligned}
$$

and the Hermitian conjugate of (30). The time derivatives of the field operators read as

$$
\begin{gathered}
\dot{\hat{\Psi}}(\mathbf{x}, t)=\frac{i}{\sqrt{2} \sqrt{V}} \sum_{\mathbf{p}} \frac{\sqrt{\Delta}}{\sqrt{\hbar \Omega(\mathbf{p}, t)}} \times \\
\times\left(-\left(\frac{\Delta / 2+\varepsilon_{C}(\mathbf{p})}{\hbar}\right) \hat{a}_{e}(\mathbf{p}) \mathrm{e}^{-i\left(\frac{\Delta / 2+\varepsilon_{C}(\mathbf{p})}{\hbar}\right) t}+\right. \\
\left.+\left(\frac{\Delta / 2+\varepsilon_{V}(-\mathbf{p})}{\hbar}\right) a_{h}(-\mathbf{p}) \mathrm{e}^{i\left(\frac{\Delta / 2+\varepsilon_{V}(-\mathbf{p})}{\hbar}\right) t}\right) \mathrm{e}^{\frac{\mathbf{p x}}{\hbar}}
\end{gathered}
$$

and the Hermitian conjugate of (31). 
The direct and Hermitian conjugate canonical momenta in operator representation are found by substituting the field operators in (10) [11]

$$
\begin{gathered}
\hat{\pi}(\mathbf{x}, t)=i \sum_{\mathbf{p}} \sqrt{\frac{\Delta \Omega(\mathbf{p}, t)}{2 \Delta V \hbar} \times} \\
\times\left(\mathrm{e}^{\frac{i}{\hbar}\left(\frac{\Delta}{2}+\varepsilon_{c}(\mathbf{p})\right) t} \hat{a}_{e}^{+}(\mathbf{p})-\mathrm{e}^{-\frac{i}{\hbar}\left(\frac{\Delta}{2}+\varepsilon_{v}(-\mathbf{p})\right) t} \hat{a}_{h}^{+}(-\mathbf{p})\right) \mathrm{e}^{-i \frac{\mathbf{p} \mathbf{x}}{\hbar}}
\end{gathered}
$$

and the Hermitian conjugate of (32) where the relation $\Delta+\varepsilon_{C}(\mathbf{p})+\varepsilon_{V}(-\mathbf{p}) \equiv 2 \hbar \Omega(\mathbf{p}, t)$ and the antisymmetric commutation relations are accounted for. Canonical quantization for field operators is obtained by replacements $\exp \left[-\frac{i}{\hbar}\left(\frac{\Delta}{2}+\varepsilon_{c}(\mathbf{p})\right) t\right] \hat{a}_{e}(\mathbf{p}) \Rightarrow \hat{a}_{e}(\mathbf{p}, t)$ etc.

Functional derivatives of the Lagrangian density in (27), (29) with respect to birth and annihilation operators $\hat{a}_{e}(\mathbf{p}, t)$, $\hat{a}_{e}^{+}(\mathbf{p}, t), \quad \hat{a}_{h}(-\mathbf{p}, t) . \hat{a}_{h}^{+}(-\mathbf{p}, t)$ and the condition $\delta S=0$ yields the set of kinetic equations as follows:

$$
\begin{gathered}
\dot{\hat{a}}_{e}(\mathbf{p}, t)=\frac{\lambda \hat{a}_{h}(-\mathbf{p}, t)}{2}-\frac{i}{2 \hbar}\left(\frac{\Delta}{2}+\varepsilon_{c}(\mathbf{p})\right) \hat{a}_{e}(\mathbf{p}, t), \\
\dot{\hat{a}}_{e}^{+}(\mathbf{p}, t)=\frac{\lambda}{2} \hat{a}_{h}^{+}(-\mathbf{p}, t)+\frac{i}{2 \hbar}\left(\frac{\Delta}{2}+\varepsilon_{c}(\mathbf{p})\right) \hat{a}_{e}^{+}(\mathbf{p}, t), \\
\dot{\hat{a}}_{h}(-\mathbf{p}, t)=\frac{\lambda}{2} \hat{a}_{e}(\mathbf{p}, t)+\frac{i}{2 h}\left(\frac{\Delta}{2}+\varepsilon_{v}(\mathbf{p})\right) \hat{a}_{h}(-\mathbf{p}, t), \\
\dot{\hat{a}}_{h}^{+}(-\mathbf{p}, t)=\frac{\lambda}{2} \hat{a}_{e}^{+}(\mathbf{p}, t)-\frac{i}{2 \hbar}\left(\frac{\Delta}{2}+\varepsilon_{v}(\mathbf{p})\right) \hat{a}_{h}^{+}(-\mathbf{p}, t),
\end{gathered}
$$

where $\lambda=\dot{\Omega}(\mathbf{p}, t) / \Omega(\mathbf{p}, t)$ is the amplitude of interband transitions [8] and each of equations (33)-(36) describe some mixture of positive and negative energy states [22].

Furthermore, in accord with the asymptotic condition, the optical radiation is switched off in the infinite past

$$
\lim _{t \rightarrow-\infty} \mathbf{E}(t)=-\lim _{t \rightarrow-\infty} \mathbf{A}(t)
$$

but, due the nondissipative nature of the Klein-Gordon approach, the final solution may be nonzero [23].

\section{Canonical quantization of acoustic phonons}

In the previous section, we have emphasized that the dynamics of the photooptically excited electron-hole pairs can be calculated on the basis of the macroscopic Hamiltonian comprising dispersion relations for electrons and holes and the lattice field constituted of the ionic subsystem. Subsequent canonical quantization yields the set of kinetic equations for birth and annihilation operators as well as the nonequilibrium distribution functions for (quasi)electrons and holes. This approach is regarded as nonperturbative, non-Markovian, applicable to variable laser pulses, and variable number of emerging optically excited electronhole pairs. However these advancements are obtained by decoupling of the dynamics of the electrons and ions and neglecting electronic excitations induced by the ionic motion. Deficiencies of this $a b$ initio Hamiltonian approach appear especially in treating strongly correlated systems, i.e., $d$ - and $f$-electron systems which have a Coulomb interaction comparable to the band width. On the other hand the most successful technique based on the DFT+DMFT-type model Hamiltonian approach is more general to calculate many-electron problems but at the same time is seriously restricted in its ability to make quantitative predictions since the input parameters are not accurately known and must be adjusted.

The scientific frontier we are faced in this section is the dynamical coupling between the electronic and phonon subsystems treated on equal kinetic quantum field basis that embody both the excitation and the relaxation of the quasiparticles. The corresponding Hamiltonian formalism for interacting between electron-hole pairs and the lattice subsystem involves equations of elasticity. With the goal of developing a kinetic quantum field theory for acoustic phonons we first take advantage of the existing free field stationary solutions for displacement vector field $[15,16]$ based on the classical Lagrangian for elastic isotropic solid. We restrict to a single mode version

$$
\ddot{u}-\left(\frac{K+\Gamma}{\rho}\right) \nabla^{2} u=0,
$$

where $\rho$ is the mass density, $K$ and $\Gamma$ are two elastic moduli, and $\mathbf{u}(\mathbf{r}, t)$ is the displacement field with normal mode(s) of the lattice. The line of approach, which we think is sufficient physical one, starts from the observation that electrons and holes are quantum objects and for the finding their interaction the phonons in terms of canonically quantized solution of elasticity is important. To this end, similarly to the electron-hole case (20), the four-Fourier transform related to displacement field is

$$
u(x)=\int \frac{d^{4} p}{(2 \pi)^{4}} \tilde{u}(p) \exp \left(-i \frac{p x}{\hbar}\right) .
$$

The decomposition starts with the displacement (20) as

$$
\begin{gathered}
u(x)=\int \frac{d p_{1} d p_{2} d p_{3}}{(2 \pi)^{3}} \times \\
\times\left(\int \frac{d \omega}{2 \pi} \delta\left(\omega^{2}-\left(\frac{K+\Gamma}{\rho}\right) \frac{\mathbf{p}^{2}}{\hbar^{2}}\right) \tilde{u}_{i}(\mathbf{p}) \exp (i \omega t)\right) \exp \left(-i \frac{\mathbf{p x}}{\hbar}\right),
\end{gathered}
$$

where, similarly to (23) and (25), the $g$-function and the $f$-function are given by relations 


$$
g(\omega, \mathbf{p})=\omega^{2}-\left(\frac{K+\Gamma}{\rho}\right) \frac{\mathbf{p}^{2}}{\hbar^{2}}
$$

and

$$
f(\omega, \mathbf{p})=\tilde{u}(p) \mathrm{e}^{i \omega t}
$$

Only positive root of the $g$-function is maintained whereas the negative root, supporting states with negative energy, are discarded as

$$
\omega(\mathbf{p})=+\sqrt{\frac{K+\Gamma}{\rho}} \frac{|\mathbf{p}|}{\hbar} .
$$

Derivative of the function $g(\omega, \mathbf{p})_{\omega}=2 \omega$ and the common denominator in the generalized scaling property reads as

$$
\left|g^{\prime}\left(\omega_{1}\right)\right|=2 \sqrt{\frac{K+\Gamma}{\rho}} \frac{|\mathbf{p}|}{\hbar} \Rightarrow 2 \omega(\mathbf{p}) .
$$

Complete relativistic quantum field approach [19] is provided equally to this in Sec. 4 and results in sum over momentum space

$$
u(x)=\frac{1}{\sqrt{\rho}} \frac{1}{\sqrt{V}} \sum_{\mathbf{p}} \frac{1}{2 \omega(\mathbf{p})} \tilde{u}(\mathbf{p}) \exp (i \omega(\mathbf{p}) t) \exp \left(i \frac{\mathbf{p x}}{\hbar}\right) .
$$

Positive energy solution [16,24] is supported by replacement of (38) to

$$
\hat{u}(x)=\frac{1}{\sqrt{V}} \sum_{\mathbf{p}} \frac{1}{2 \omega(\mathbf{p})}\left[\hat{b}_{\mathbf{p}}(\mathbf{p}) \mathrm{e}^{i \omega(\mathbf{p}) t} \mathrm{e}^{-i \frac{\mathbf{p x}}{\hbar}}+\hat{b}_{\mathbf{p}}^{+} \mathrm{e}^{-i \omega(\mathbf{p}) t} \mathrm{e}^{i \frac{\mathbf{p x}}{\hbar}}\right],
$$

where the stationary birth and annihilation operators $\hat{b}_{\mathbf{p}}^{+}(\mathbf{p})$ and $\hat{b}_{\mathbf{p}}(\mathbf{p})$ follow in conventional way.

Going to the quantum kinetics in a simplest way, the impact of electronic subsystem is accounted for by implementing time dependence in the $g$-function (43) as a parameter $\omega(\mathbf{p}, t)$ so transforming (37) to the differential equation with variable coefficients. In a simpler case, making use of the quantum kinetics of electron-hole pairs, the kinetic equations for operators $\hat{b}_{\mathbf{p}}^{+}(\mathbf{p}, t)$ and $\hat{b}_{\mathbf{p}}(\mathbf{p}, t)$ support terms $\dot{\omega}(\mathbf{p}, t) / \omega(\mathbf{p}, t)$ as the measure of the interaction.

At this point there is an immediate obstacle: there is not a satisfactory quantum field approach for time-dependent operators $\hat{b}_{\mathbf{p}}^{+}(\mathbf{p}, t)$ and $\hat{b}_{\mathbf{p}}(\mathbf{p}, t)$ in systems with only positive energy allowed [16,24] restricted within the scalar Klein-Gordon field.

The generalization to the desired kinetic theory making use of the same type of the complex Klein-Gordon field (20) is left to a separate publication.

\section{Conclusions}

In this paper, we developed a general theory of photooptical properties of semiconductors and dielectrics under strong electromagnetic (laser) radiation at low temperatures. The physical model includes spatially periodic structure of atoms supporting quasiparticles categorized as electrons, holes and acoustic phonons, each having a complex influence on its neighbors.

The approach merges the birth and annihilation of the charged quasiparticles (electrons and holes) with the acoustic phonons. To solve this chalenging problem, we used the methods of quantum electrodynamics which contribute to the consistent relativistic theory of open quantum systems. In terms of quantum electrodynamics, we have considered both the complex and the scalar Klein-Gordon field for the electron-hole pairs and the displacement field of phonons.

The illustrative solutions are obtained by the transition from the macroscopic description to the quantum field formalism and kinetic equations for the constituting quasiparticles. Corresponding distribution functions and observables of interest are beyond scope of this manuscript. The insights gained in this study could contribute to fundamental understanding of the complex joint wave functions and the higher order energy derivatives in strongly correlated condensed matter systems.

The partial support by Latvian research program IMIS2 is highly appreciated.

1. A. Georges, G.l. Kotliar, W. Krauth, and M.J. Rozenberg, Rev. Modern Phys. 68, 13 (1996).

2. F. Lechermann, L. Boehnke, and D.Grieger, Phys. Rev. B 87, 241101 (2013).

3. D. Vollhardt, K. Byczuk, and M. Kollar, Dynamical MeanField Theory, Chapter in: Theoretical Methods for Strongly Correlated Systems, A. Avella and F. Mancini (eds.), Springer (2011).

4. J. Schwinger, Phys. Rev. 82, 664 (1951).

5. S. Schmidt, D. Blaschke, G. Röpke, A.V. Prozorkevich, S.A. Smolyansky, and V.D. Toneev, Phys. Rev. D 59, 094005 (1999).

6. V.N. Pervushin and V.V. Skokov, Acta Phys. Polonica B 37, No. 9, 2587 (2006).

7. D.B. Blasche, D.B. Blaschke, B. Kämpfer, S.M. Schmidt, A.D. Panferov, A.V. Prozorkevich, and S.A. Smolyansky, Phys. Rev. D 88, 045017 (2013).

8. A.V. Filatov, A.V. Prozorkevich, S.A. Smolyansky, and V.D. Toneev, Phys. Part. Nucl. 39, 886 (2008).

9. A.V. Friesen et al., Proc. SPIE 6537, Laser Physics and Photonics, Spectroscopy and Molecular Modeling VII, 653 (2007).

10. S.A. Smolyansky, A.V. Tarakanov, and M. Bonitz, Contrib. Plasma Phys. 49, 575 (2009).

11. S.A. Smolyansky, M. Bonitz, and A.V. Tarakanov, Phys. Particl. Nucl. 41, 1075 (2010). 
12. A.V. Kimel and A.K. Zvezdin, Fiz. Nizk. Temp. 41, 878 (2015) [Low Temp. Phys. 41, 682 (2015)].

13. Y.V. Skripnik, Fiz. Nizk. Temp. 41, 501 (2015) [Low Temp. Phys. 41, 390 (2015)].

14. N.E. Sluchanko, Fiz. Nizk. Temp. 41, 699 (2015) [Low Temp. Phys. 41, 544 (2015)].

15. S. Fujita, K. Ito, and S. Godoy, Quantum Theory of Conducting Matter, Spinger, LLC (2009).

16. Chetan Nayak, Many-Body Physics, University of California, Los Angeles (1999).

17. Mark Thomson, Modern Particle Physics, Cambridge University Press (2013).

18. L. Álvarez-Gaumé and M.Á. Vázques-Mozo, An Invitation to Quantum Field Theory, Lecture Notes in Physics 839, Spriger-Verlag, Berlin-Heidelberg (2012).
19. Michael A. Nielsen and Isaac L. Chuang, Quantum Computation and Quantum Information, Cambridge University Press (2000).

20. E. Klotins, IOP Conf. Series: Materials Science and Engineering 77, 012003 (2015).

21. J.D. Walecka, Topics in Modern Physics, Theoretical Foudations, World Scientific Publishing (2013).

22. V.N. Pervushin, V.V. Skokov, A.V. Reichel, S.A. Smolyansky, and A.V. Prozorkevich, Int. J. Mod. Phys. A 20, 5689 (2005).

23. V.S. Popov and M.S. Marinov, Phys. Atom Nuclei 16, 809 (1972).

24. J.W. Norbury, Quantum Field Theory, Physics Department, University of Wisconsin-Milwaukee (2000). 